\title{
CORRECTION
}

\section{Correction to: Efficient fast motion estimation algorithm for real-time applications}

\author{
Shahrukh Agha' ${ }^{1}$ (D) Mansoor Khan ${ }^{1} \cdot$ Farmanullah Jan ${ }^{2}$
}

Published online: 7 January 2022

○) Springer-Verlag GmbH Germany, part of Springer Nature 2021

Correction to: Journal of Real-Time Image Processing https://doi.org/10.1007/s11554-021-01188-7

Publisher's Note Springer Nature remains neutral with regard to jurisdictional claims in published maps and institutional affiliations.

The acknowledge section is missing in the original article. The acknowledgement text is given in this erratum.

\section{Acknowledgement}

Authors are thankful to the Deanship of Scientific Research (DSR), Imam Abdulrahman Bin Faisal University (IAU) for supporting this work under project numbered 2019-381-CSIT.

The original article has been corrected.

The original article can be found online at https://doi.org/10.1007/ s11554-021-01188-7.

Shahrukh Agha

shahrukh_agha@comsats.edu.pk

1 Department of Electrical and Computer Engineering, COMSATS University Islamabad, Islamabad 44000, Pakistan

2 Department of Computer Science, Imam Abdulrahman Bin Faisal University, Dammam, Saudi Arabia 